\title{
Phonons Are Key in Strained Superconductors
}

\author{
Substrate-induced strain in $\mathrm{RuO}_{2}$ thin films generates the kind of phonons \\ that promote superconductivity.
}

By Marric Stephens

$\sum^{2}$ ecent technological advances have allowed researchers to make exceptionally high-quality, thin, metal oxide films, spurring new experiments. One such experiment revealed superconducting behavior in a roughly 30 -nm-thick layer of ruthenium oxide $\left(\mathrm{RuO}_{2}\right)$-but only when it was grown on a suitably oriented titanium dioxide $\left(\mathrm{TiO}_{2}\right)$ substrate. Now, Masaki Uchida at the University of Tokyo and colleagues have identified the cause of this behavior [1]. Their finding could provide a starting point for inducing superconductivity in other metal oxide films.

When a thin metal oxide film is grown on a substrate with different lattice parameters, the mismatch creates stresses that deform the bonds between the film's atoms. This process was known to promote superconductivity in $\mathrm{RuO}_{2}$, but the mechanism behind this promotion was unclear. To investigate, the Tokyo team grew $\mathrm{RuO}_{2}$ thin films on either a $\mathrm{TiO}_{2}$ substrate or a magnesium fluoride $\left(\mathrm{MgF}_{2}\right)$ substrate with various crystal orientations and then measured the $\mathrm{RuO}_{2}$ 's conductivity as the films were cooled to $0.4 \mathrm{~K}$.

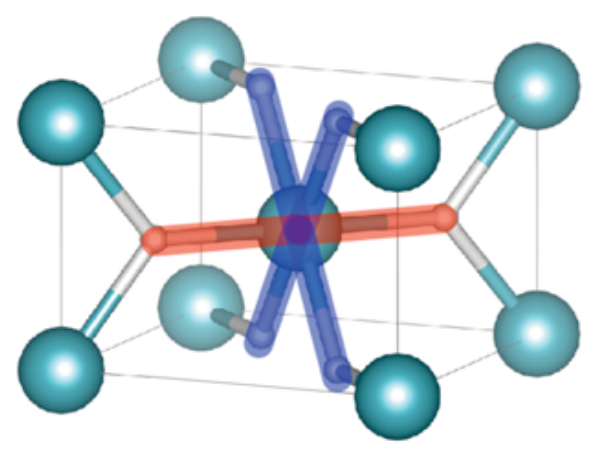

Credit: M. Uchida et al. [1]
The team found that only one sample superconducted: the $\mathrm{RuO}_{2}$ film grown on 110 -oriented $\mathrm{TiO}_{2}$, which started superconducting at $1.7 \mathrm{~K}$. In that sample, the lattice mismatch squeezed a specific metal-oxygen bond in the $\mathrm{RuO}_{2}$ 's crystal structure. Using density-functional calculations, the researchers found that the shortening of this bond lowered the frequency of phonons traveling along the unit cell's long axis. Such "soft" phonons are known to deform the ion lattice, promoting superconductivity in $\mathrm{RuO}_{2}$ by creating the electronic environment necessary for electrons to pair up and propagate without resistance. But, the researchers say, why phonons traveling in this particular direction are so important remains unclear.

Marric Stephens is a Corresponding Editor for Physics based in Bristol, UK.

\section{REFERENCES}

1. M. Uchida et al., "Superconductivity in uniquely strained $\mathrm{RuO}_{2}$ films," Phys. Rev. Lett. 125, 147001 (2020). 